\title{
A Simple Equivalent Circuit for Efficiency Calculation of Brushless DC Motors
}

\author{
Takeo Ishikawa *, Takuma Tsuji **, Seiji Hashimoto **, and Nobuyuki Kurita **
}

\begin{abstract}
This paper shows a calculation method of several types of loss and the efficiency of brushless DC motors coupled with a load system by using a simple equivalent circuit, in which copper loss, eddy current loss, hysteresis loss, friction loss, viscous loss, and inverter loss are taken into account. We clarify each loss and motor efficiency at different motor speeds and different output torques by using the Microsoft-Excel. Moreover, the calculated results are in good agreement with the measured ones.
\end{abstract}

\section{Keywords: Brushless DC motor, Equivalent circuit, Loss, Efficiency}

\section{Introduction}

Brushless DC motors are widely used in industrial applications and computer peripheral devices, because of no brush and no commutator, wide speed range, and relatively high efficiency. When the motor is connected to a load system, there are several types of loss, namely, copper loss, eddy current loss, hysteresis loss in the motor, and friction loss and viscous loss in the motor and load system, and inverter loss. These losses change according to the operating speed and the load condition. For example, when the motor speed becomes high, the iron loss increases, which reduces the motor performance. Hence, the analysis and prediction of numerous types of loss in the motors are essential. The conventional analysis method used to assess the iron loss especially the eddy-current loss is a threedimensional finite element method [1]-[4]. However this method is very time consuming. It is important to calculate easily the motor efficiency at different operating speeds and load conditions. An equivalent circuit method is very useful for easy calculation of the losses and efficiency of the motor. A resistance corresponding to eddy-current loss was introduced in the $\mathrm{d}$ - and q-axis equivalent circuits [5]. An equivalent circuit for DC motor considering numerous types of losses was proposed, and the relationship between the maximum current and the currents at no-load and locked conditions was expressed when the equivalent circuit was applied to calculate the brushless DC motors [6].

\footnotetext{
* Division of Electronics and Informatics, Faculty of Science and Technology, Gunma University, Japan. (ishi@el.gunma-u.ac.jp)

** Division of Electronics and Informatics, Faculty of Science and Technology, Gunma University, Japan.

Received 30 October 2013; Accepted 1 December 2013
}

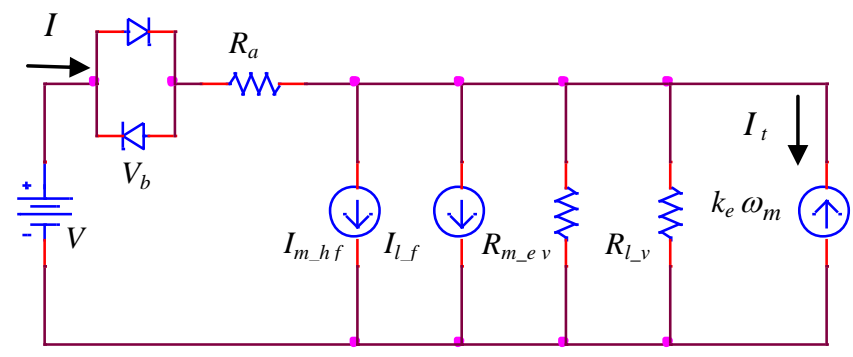

Fig. 1. A simple equivalent circuit for brushless DC motors.

This paper proposes a simple equivalent circuit for the calculation of losses and efficiency of brushless DC motors. The equivalent circuit is an improved one, which is described in [6]. This paper shows how to decide the parameters of the simple equivalent circuit for copper loss, eddy current loss and hysteresis loss in the motor, friction loss and viscous loss in the mechanical system, and voltage-drop loss in the inverter circuit. This paper clarifies each loss and the efficiency of the brushless DC motor at different motor speeds and different output torques using the Microsoft-Excel. Moreover, the comparison with experimental results is discussed.

\section{Equivalent Circuit}

\subsection{Equivalent Circuit for Brushless DC motors}

A simple equivalent circuit for brushless DC motors is shown in Fig. 1. A well-known steady state equivalent circuit for DC motors is given by the input voltage $V$, the armature resistance $R_{a}$, and the electromotive force $k_{e} \omega_{m}$. This paper proposes to improve it for brushless DC motors. $V$ and $I$ are the input voltage and input current to the 


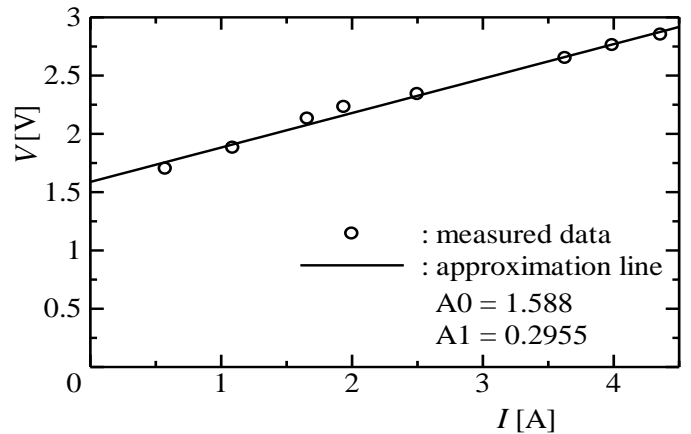

Fig. 2. $V-I$ characteristic at the lock test.

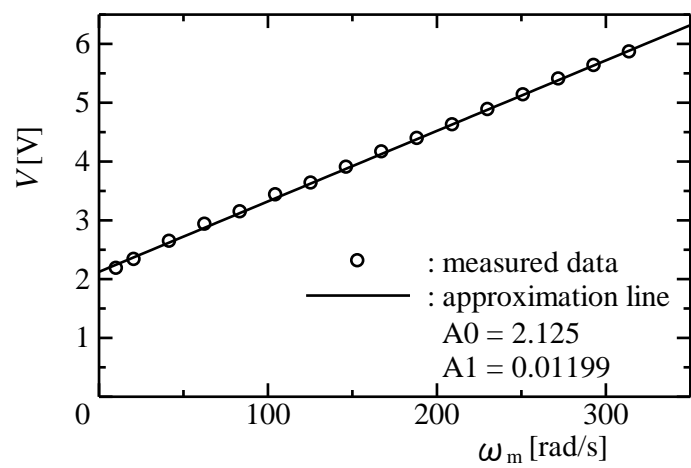

Fig. 3. $V-\omega_{m}$ characteristic at the no-load test.

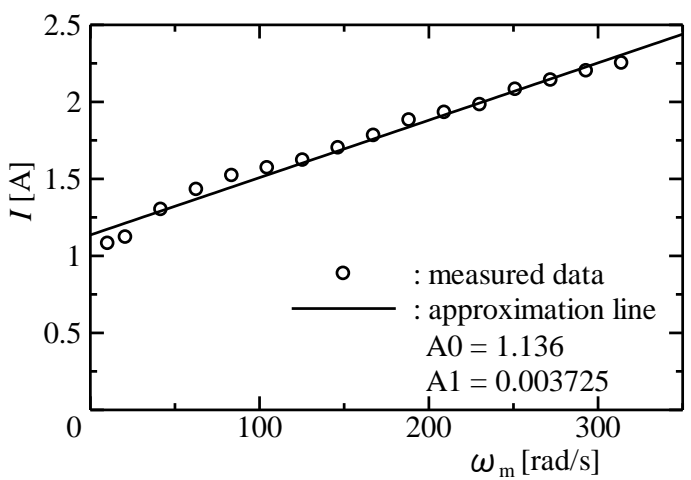

Fig. 4. $I-\omega_{m}$ characteristic at the no-load test.

Inverter, respectively. The voltage drop of transistor and diode in the inverter circuit is introduced by a forward voltage $V_{b}$ of two diodes connected in reversely parallel. The resistance of transistor and diode is also included into $R_{a}$, namely, $R_{a}$ is the summation of stator resistance of the motor and the resistance of transistor and/or diode. Eddy current loss is proportional to the square of the motor speed, and the mechanical viscous loss is also proportional to the square of the motor speed. Hence, the loss can be represented by resistances $R_{m_{-} e v}$ and $R_{l_{-} v} . R_{m_{-} e v}$ corresponds to eddy current loss plus viscous loss of the motor, and $R_{l_{-} v}$ corresponds to viscous loss of the load system. Hysteresis loss is proportional to the motor speed, and the mechanical friction loss is also proportional to the motor speed. Hence, the losses can be represented by current sources $I_{m_{-} h f}$ and $I_{l_{-}}$.
$I_{m \_h f}$ corresponds to hysteresis loss plus friction loss of the

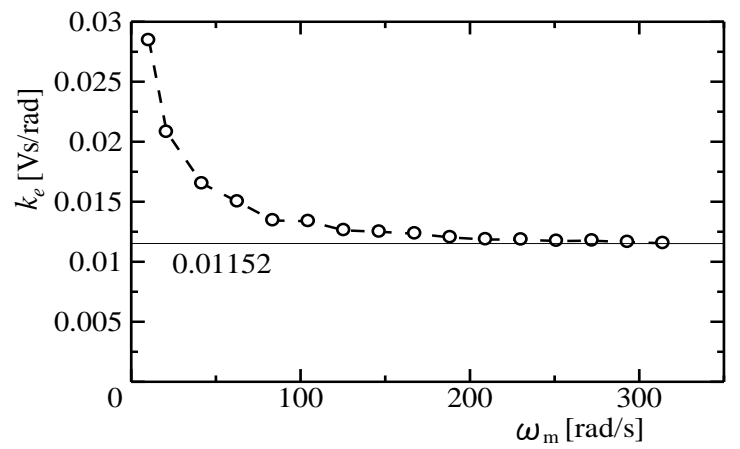

Fig. 5. $k_{e}-\omega_{m}$ characteristic.

motor, and $I_{l \_f}$ corresponds to friction loss of the load system. They are connected in parallel to $k_{e} \omega_{m}$.

\subsection{Measurement of Parameters in Equivalent Circuit for Brushless DC Motor}

Parameters in the circuit can be decided by two simple experiments. One is a lock test. When the motor speed $\omega_{m}$ is 0 , the input voltage is given by

$$
V=\left|V_{b}\right|+R_{a} I \text {. }
$$

Therefore, $V_{b}$ and $R_{a}$ can be decided by $V$ - $I$ characteristic. Fig. 2 shows the $V-I$ characteristic of the lock test. Using the approximation line shown in this figure, two parameters are estimated as $R_{a}=0.2955 \Omega, V_{b}=1.588 \mathrm{~V}$.

The other is a no-load test. When the output power is 0 , the current flowing through $k_{e} \omega_{m}$ is 0 . Hence, the input voltage and current are given by

$$
\begin{gathered}
V=\left|V_{b}\right|+R_{a} I+k_{e} \omega_{m} \\
I=k_{e} \omega_{m} /\left(R_{m_{-} e v} / / R_{l_{-} v}\right)+I_{m_{-} h f}+I_{l_{-} f} .
\end{gathered}
$$

Therefore, $k_{e}$ can be decided by $V-\omega_{m}$ characteristic, and $\left(R_{m_{-} e v} / / R_{l_{-} v}\right)$ and $\left(I_{m_{-} h f}+I_{l_{-} f}\right)$ can be decided by $I-$ $\omega_{m}$ characteristic. Figs. 3 and 4 show $V-\omega_{m}$ and $I-\omega_{m}$ characteristics, when the motor coupled with a brake is rotating without developing output torque. We estimate that $\left(R_{m_{-} e v} / / R_{l_{-} v}\right)=3.108 \Omega$ and $\left(I_{m_{-} h f}+I_{l_{-} f}\right)=1.136 \mathrm{~A}$. We also measured the $V-\omega_{m}$ and $I-\omega_{m}$ characteristics, when the motor is rotating without the brake, that is, motor alone. From these experiments, we obtained

$$
\begin{aligned}
& R_{m_{-} e v}=6.03 \Omega \\
& R_{l_{-} v}=10.59 \Omega \\
& I_{m_{-} h f}=0.378 \mathrm{~A} \\
& I_{l_{-} f}=0.758 \mathrm{~A} .
\end{aligned}
$$

$k_{e}$ is given as a function of $\omega_{m}$ as shown in Fig. 5. In order to check $k_{e}$, we measured the electromotive force (EMF) of 
the motor. Fig. 6 shows the waveform of EMF and the

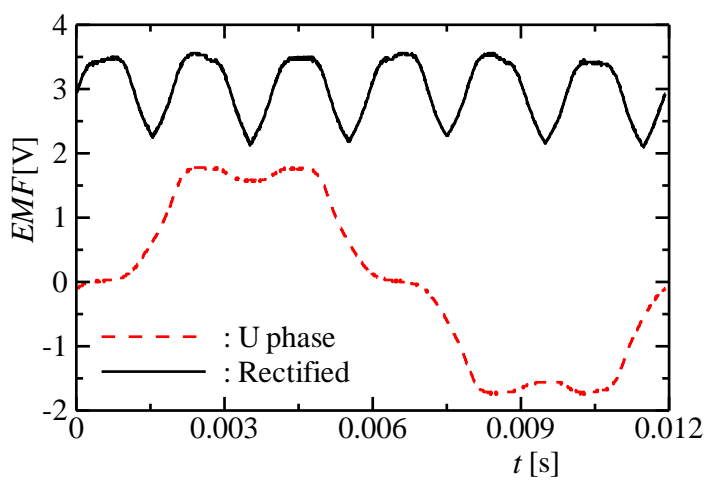

Fig. 6. Electromotive force of the experimental motor.

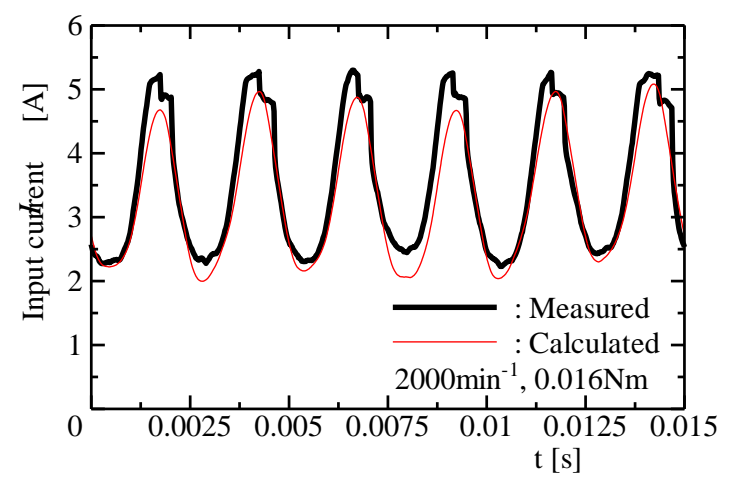

Fig. 7. Measured and calculated input currents.

rectified waveform of three-phase EMF. The mean value of the rectified EMF gives $k_{e}=0.01152[\mathrm{Vs} / \mathrm{rad}]$. It is found from Fig. 5 the value of $k_{e}$ calculated by the no-load test approaches to the value given by EMF.

It is also found that the EMF includes large ripple. This ripple produces a ripple in the input current as shown in Fig. 7. The ripple in the input current increases the copper loss and the eddy current loss. Fig. 8 shows an equivalent circuit for the calculation of ripple current. In this figure, $V, V_{b}$, $I_{m \_h f}, I_{l \_f}$ and $R_{l_{\_} v}$ are omitted, and $k_{e} \omega_{m}$ is replaced by $\Delta E$ and $L$ is inserted. Here, $\Delta E$ is the harmonic components of EMF, and $L$ is the inductance between two terminals of the motor. The measured value of $L$ is $84.5 \mu \mathrm{H}$. We can take into account the copper loss and the eddy current loss in the motor by the harmonic components of EMF by using the equivalent circuit. Note that hysteresis loss by the harmonic components of EMF is not considered. It means that the area of minor magnetic hysteresis loop is 0 .

\section{Motor Characteristics Using Equivalent Circuit}

\subsection{Loss and Efficiency}

When the torque $T_{e}$ and the motor speed $\omega_{m}$ are specified as independent variables, the input voltage $V$, the

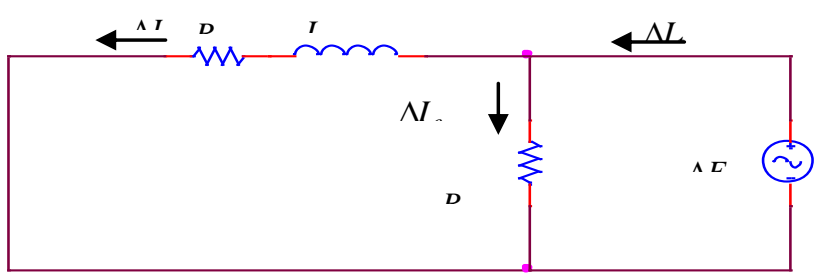

Fig. 8. Equivalent circuit for the calculation of ripple component.

input current $I$, the output power $P_{o}$, the copper loss $W_{c}$, the eddy current and mechanical viscous loss $W_{e}$, the hysteresis and mechanical friction loss $W_{h}$, the voltage-drop loss of transistor and diode $W_{t}$, and the efficiency $\eta$ are given by as follows using the simple equivalent circuit,

$$
\begin{aligned}
& I_{t}=T_{e} / k_{e} \\
& I=k_{e} \omega_{m} /\left(R_{m_{-} e v} / / R_{l_{-} v}\right)+I_{m_{-} h f}+I_{l_{-} f}+I_{t} \\
& P_{o}=T_{e} \omega_{m} \\
& W_{c}=R_{a} I^{2} \\
& W_{e}=\left(k_{e} \omega_{m}\right)^{2} /\left(R_{m_{-} e v} / / R_{l_{-} v}\right) \\
& W_{h}=k_{e} \omega_{m}\left(I_{m_{-} h f}+I_{l_{-} f}\right) \\
& W_{t}=V_{b} I \\
& \eta=P_{o} /\left(P_{o}+W_{c}+W_{t}+W_{e}+W_{h}\right) .
\end{aligned}
$$

If the ripple of EMF is taken into account, the following components should be included

$$
\begin{aligned}
& \Delta T_{e}=-\sum_{i=1}^{\infty} \operatorname{Re}\left(\Delta E_{i} \Delta I_{i}^{*}\right) \\
& \Delta W_{c}=\sum_{i=1}^{\infty} R_{a}\left(\Delta I_{i}\right)^{2} \\
& \Delta W_{e}=\sum_{i=1}^{\infty} R_{m_{-} e v}\left(\Delta I_{e_{-} i}\right)^{2}
\end{aligned}
$$

Therefore, the efficiency including the ripple of EMF is given by

$$
\begin{aligned}
& \eta^{\prime}=\left(T_{e}+\Delta T_{e}\right) \omega_{m} \\
& \quad /\left(\left(T_{e}+\Delta T_{e}\right) \omega_{m}+W_{c}+\Delta W_{c}+W_{t}+W_{e}+\Delta W_{e}+W_{h}\right)
\end{aligned}
$$

Maps of each variable are calculated by Microsoft-Excel, and are drawn in Fig. 9, where the vertical axis is not $T_{e}+\Delta T_{e}$ but $T_{e}$ for easy drawing.

\subsection{Comparison with Measured Data}

We have measured input voltage, input current, torque, motor speed and input power of an experimental motor of $100 \mathrm{~W}, 12 \mathrm{~V}$ and $2,500 \mathrm{~min}^{-1}$. Maps of each variable are drawn in Fig. 10. By comparing the input voltages shown in 


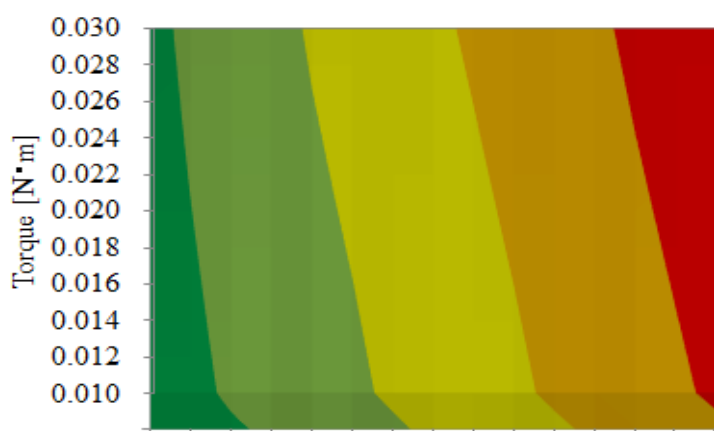

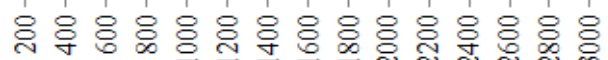
Motor speed [ $\mathrm{min}^{-1}$ ]

(a) Input voltage $V$

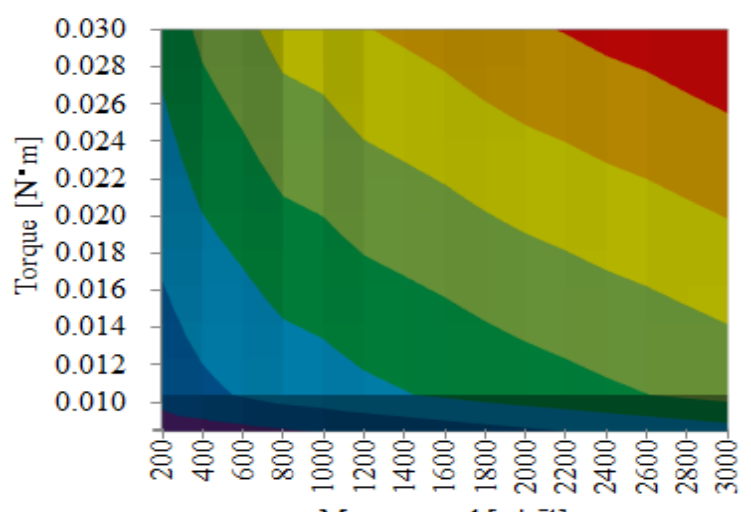

Motor speed [ $\left.\mathrm{min}^{-1}\right]$

(b) Input current $I$

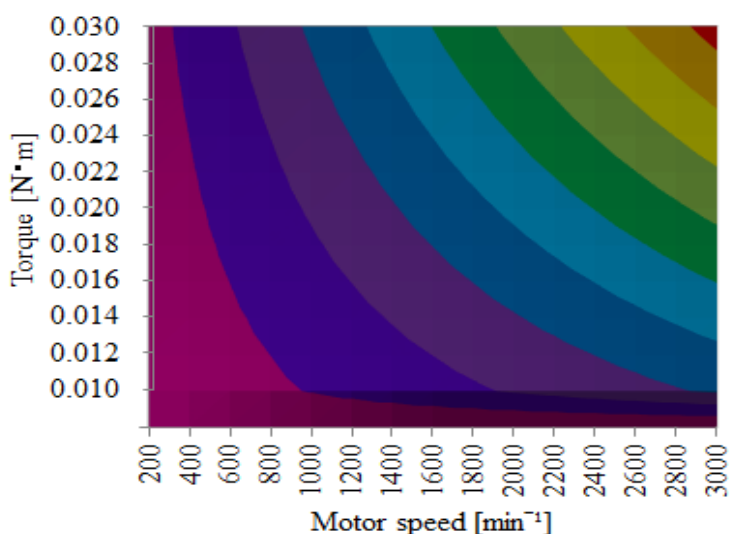

(c) Output power $P_{o}$

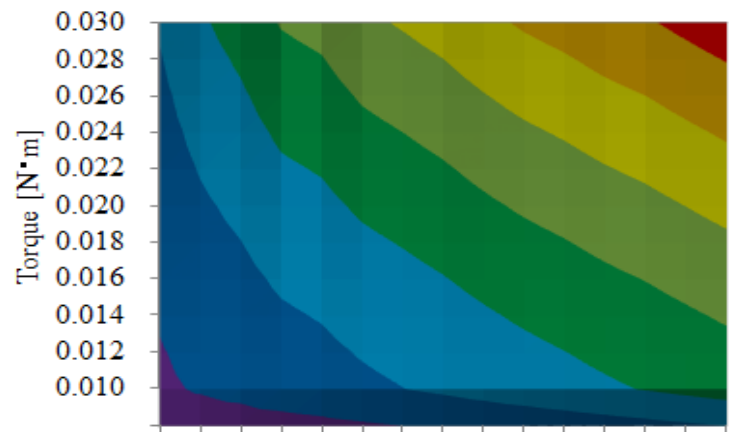

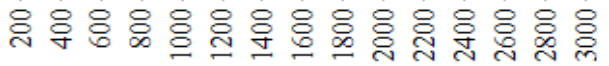
Motor speed [ $\left.\mathrm{min}^{-1}\right]$

(d) Copper loss $W_{c}$

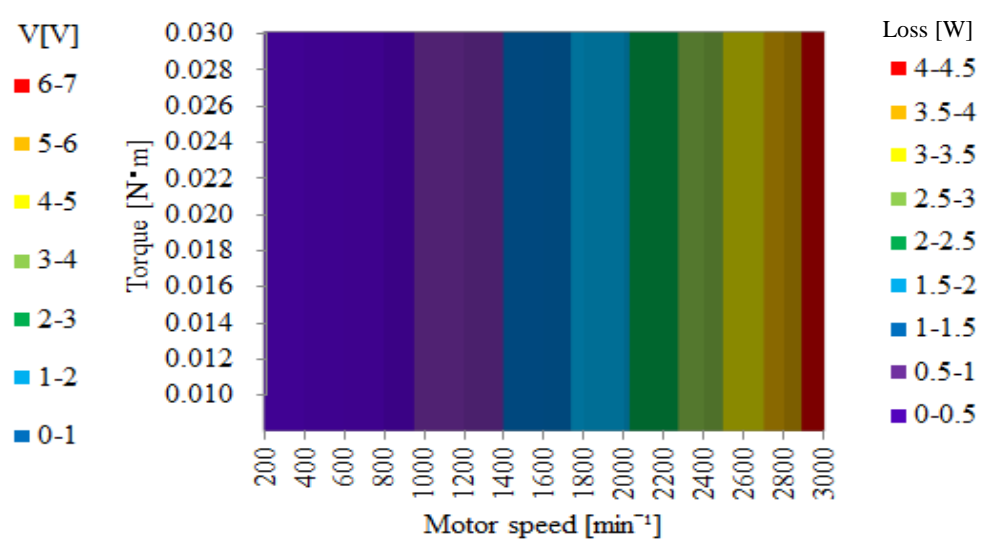

I [A] 0.031

- 4.5-5 0.028

$4-4.5-0.026$

$3.5-4 \equiv 0.024$

$=3-3.5 \div 0.022$

- $2.5-3$ 辛 0.018

E $2-2.5 \stackrel{5}{=} 0.016$

- $1.5-2 \quad 0.014$

$\begin{array}{ll}1.5-2 & 0.012\end{array}$

- 1-1.5 0.010

- $0.5-1$

- $0-0.5$

(e) Eddy current and mechanical viscous loss $W_{e}$

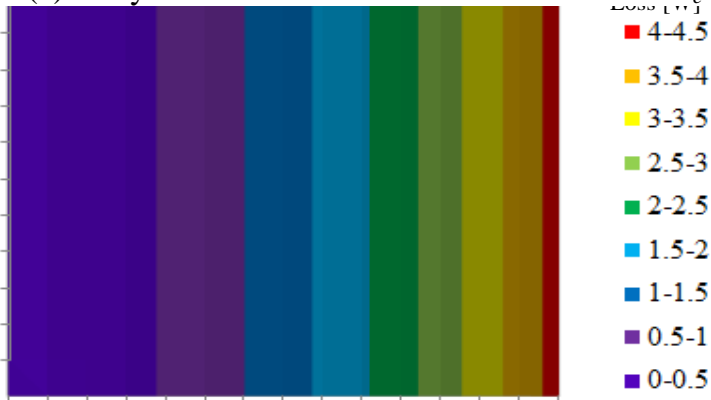

Output $\quad 0.030$

power [W]

- 9-10

- 8-9

7-8

6-7

- 5-6

- 4-5

- 3-4

a 2-3

- 1-2

- $0-1$

0.028

0.026

छ 0.024

$\Xi 0.022$

实 0.020

产 0.018

0.016

0.014

0.012

0.010

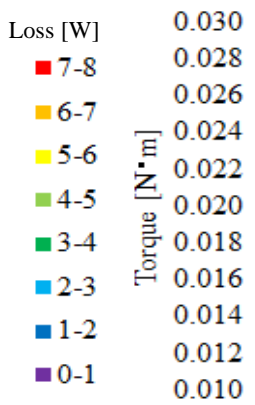

0.010

(h) Entire loss

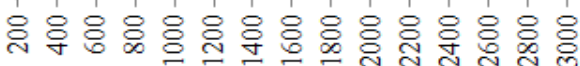

Motor speed $\left[\mathrm{min}^{-1}\right]$

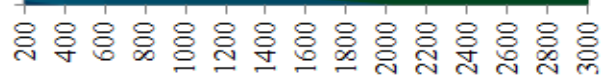

Motor speed [ $\mathrm{min}^{-1}$ ]

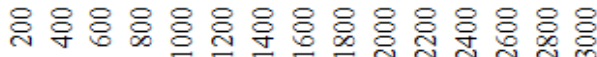

Motor speed [ $\mathrm{min}^{-1}$ ]

4-4.5

3-3.5

$2.5-3$

$2-2.5$

$1.5-2$

$0.5-1$

- $0-0.5$

(g) Voltage drop loss of transistor and diode $W_{t}$

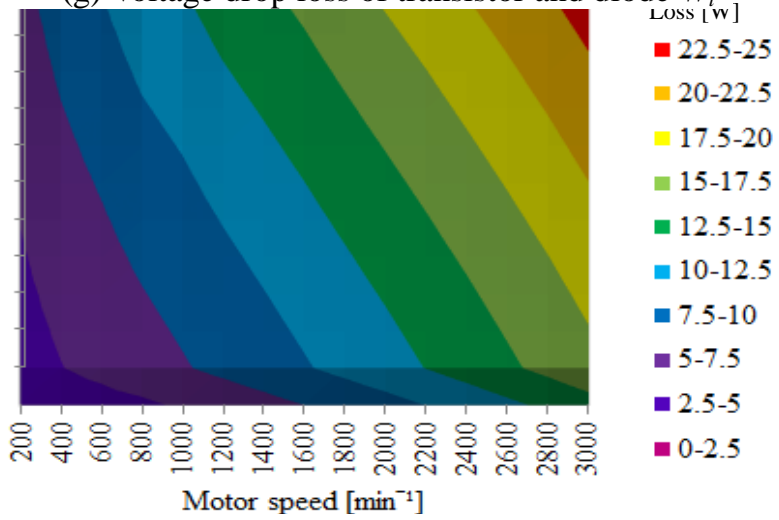

22.5-25

- 20-22.5

17.5-20

15-17.5

12.5-15

$10-12.5$

7.5-10

5-7. 


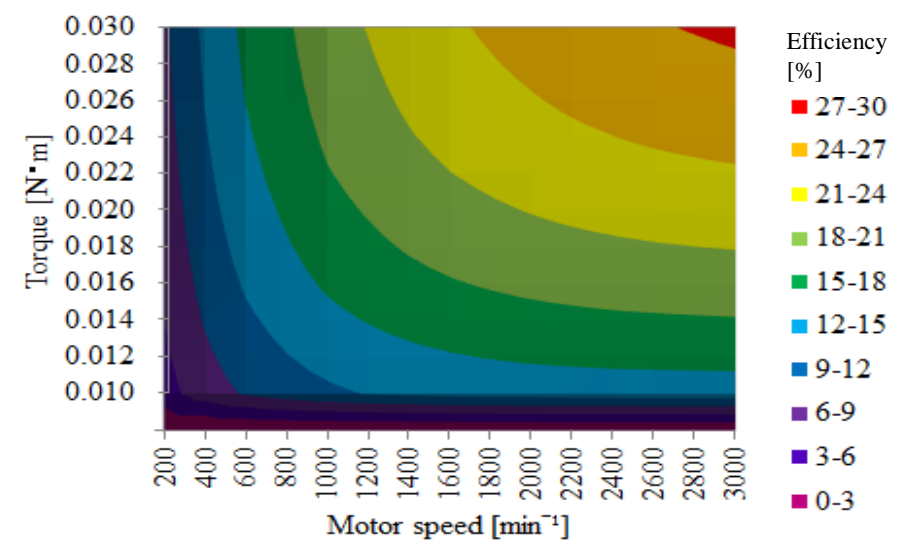

(i) Efficiency $\eta$

Fig. 9. Maps for each variable.

Figs. 9(a) and 10(a), they show vertical stripes, and the maximum difference is $0.18 \mathrm{~V}$. The input currents drawn in Figs. 9(b) and 10(b) show sloped stripes, and the maximum difference is $1.2 \mathrm{~A}$. The entire loss also shows sloped stripes, and the maximum difference is $3.6 \mathrm{~W}$. The efficiency drawn in Figs. 9(i) and 10(d) shows striped pattern of hyperbola, and the maximum difference is $2.7 \%$. Therefore, it is verified that the motor characteristics calculated by the equivalent circuit shown in Fig. 1 are in good agreement with the measured ones.

\subsection{Comparison with Conventional Equivalent Circuit}

The well-known steady state equivalent circuit for DC motors is represented by the armature resistance $R_{a}$ and the electromotive force $k_{e} \omega_{m}$, whose values are the same as those shown in Fig. 1 , namely, $V_{b,} R_{m \_e v}, R_{l_{-} v} . R_{m \_e v}, I_{m \_h f}$ and $I_{l_{-} f}$ are ignored. Maps of each variable calculated by the conventional circuit are shown in Figs. 11. The input current and the copper loss, which is equal to the entire loss give perfectly horizontal stripes. The motor efficiency give a higher value because the other losses are ignored.

\section{Simulation of Voltage and Current waveforms}

The efficiency of the experimental brushless DC motor is low as shown above, because the loss of an experimental inverter, a torque meter and a hysteresis brake is larger than the loss of the experimental motor. For example, since the stator resistance of the experimental motor is $30 \mathrm{~m} \Omega$, the resistance of transistor and/or diode, which is $0.296-0.030=$ $0.266 \Omega$, is larger than the stator resistance.

In order to verify the stator voltage and current in the system with large inverter loss, we calculate their waveforms. The voltage equations for the brushless DC

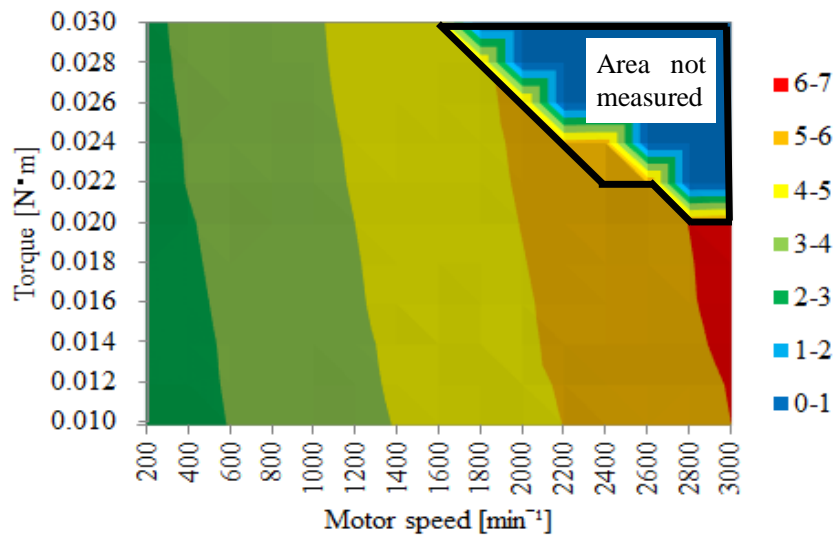

(a) Input voltage $V$

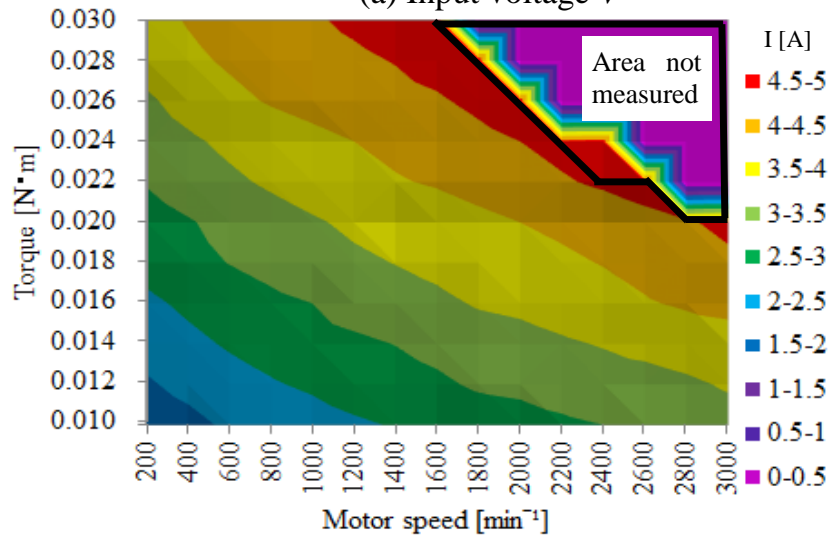

(b) Input current $I$

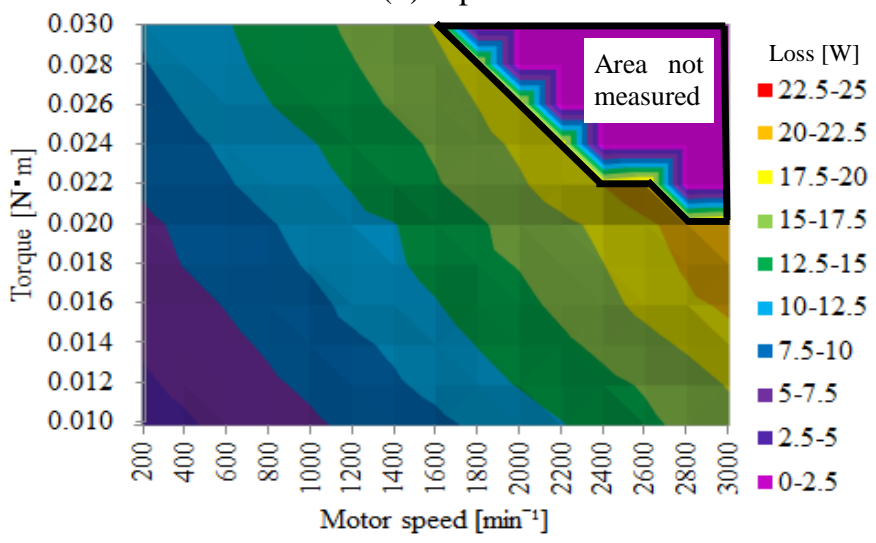

(c) Entire loss

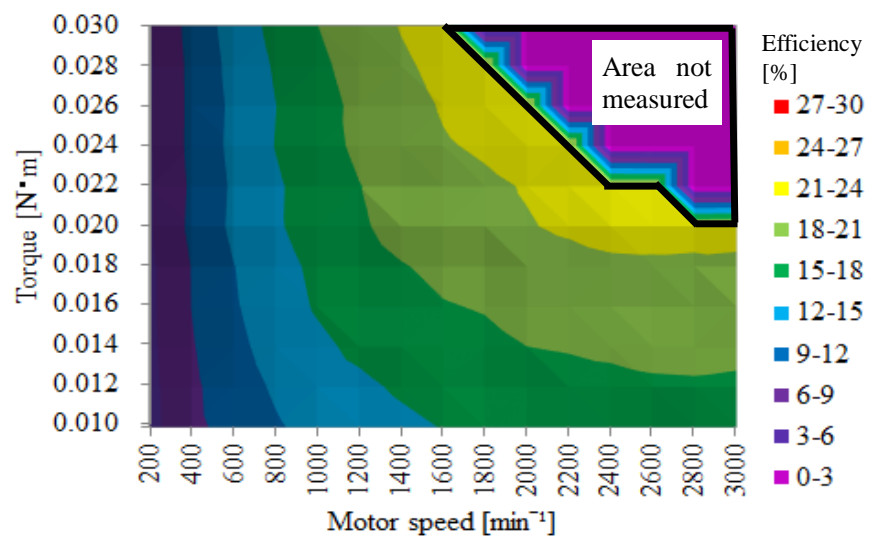

(d) Efficiency $\eta$

Fig. 10. Maps of the measured variable. 


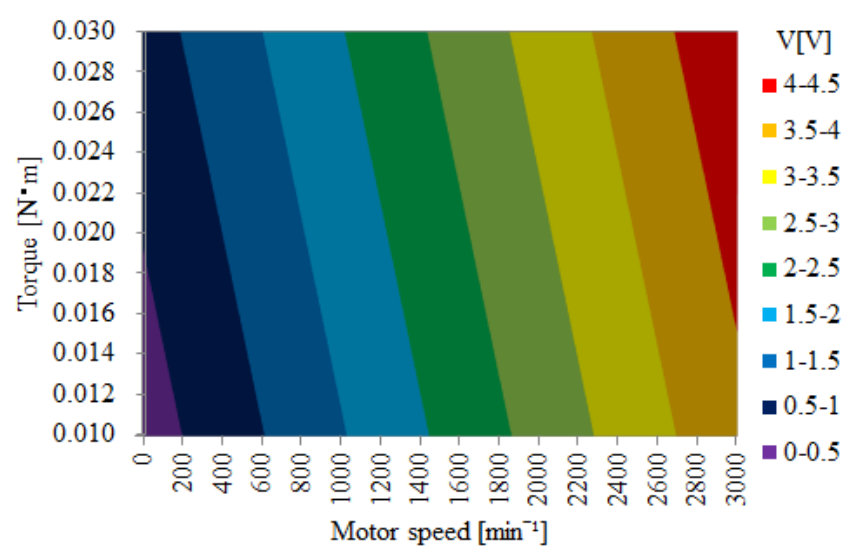

(a) Input voltage $V$

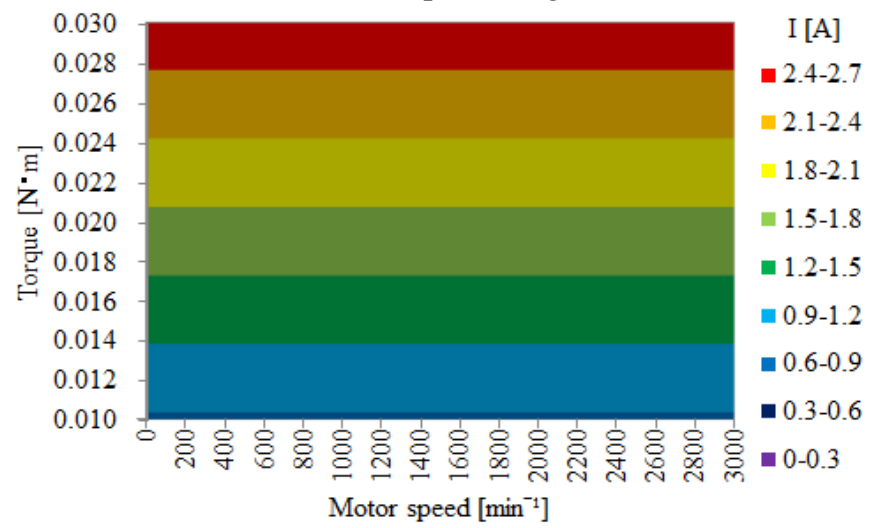

(b) Input current $I$

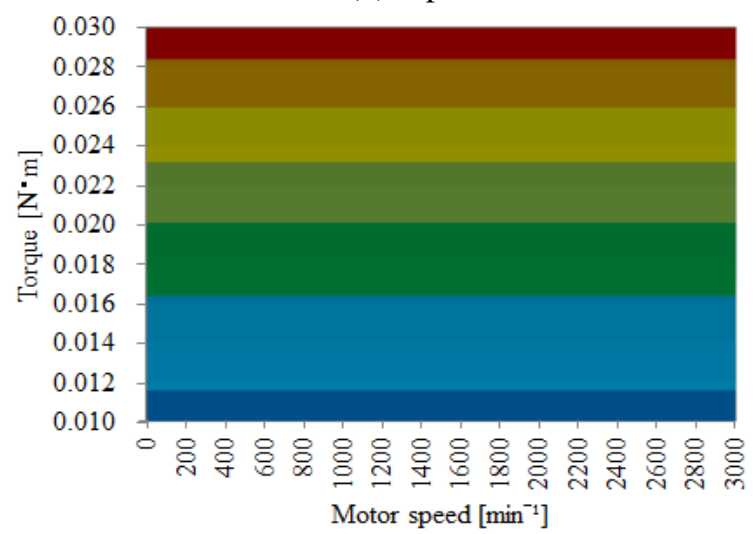

(c) Entire loss

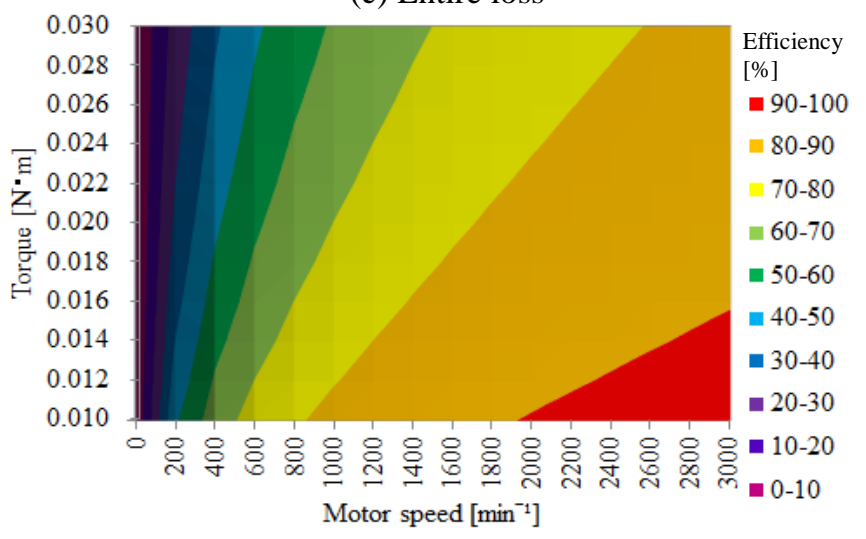

(d) Efficiency $\eta$

Fig. 11. Maps calculated with conventional equivalent

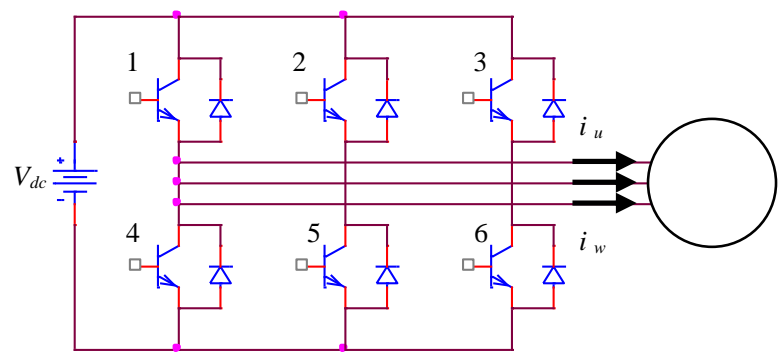

Fig. 12. Drive circuit for brushless DC motors.

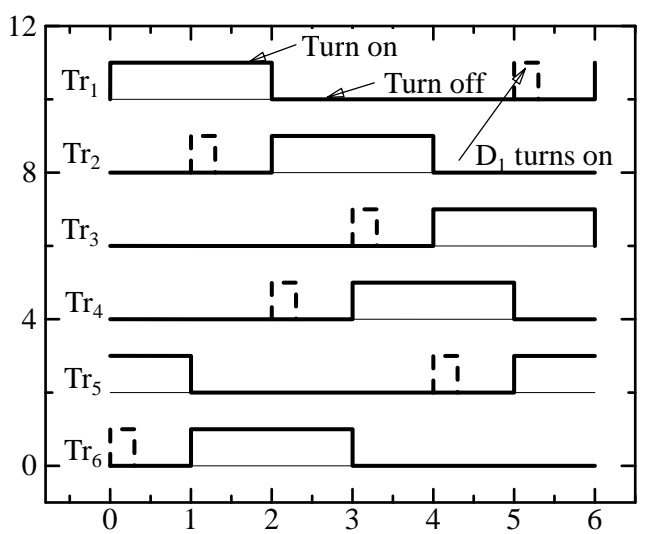

Fig. 13. Turn-on signal of each transistor.

motor shown in Fig. 12 are represented as follows by considering the summation of three stator currents $i_{u}+i_{v}+i_{w}=0$,

$$
\begin{aligned}
& (L-M) \frac{d i_{u}}{d t}+R_{a} i_{u}=1 / 3\left(-2 e_{u}+e_{v}+e_{w}+2 V_{u}-V_{v}-V_{w}\right) \\
& (L-M) \frac{d i_{v}}{d t}+R_{a} i_{v}=1 / 3\left(e_{u}-2 e_{v}+e_{w}-V_{u}+2 V_{v}-V_{w}\right)
\end{aligned}
$$

where $L, M$ and $e$ are the self-inductance, mutual inductance, and the electromotive force. The stator voltage $V_{u}$ is expressed by considering the voltage drop and the resistance of transistor and diode $V_{t r}, V_{d}, R_{t r}$ and $R_{d}$, when the turn-on signal of an upper transistor 2 is active as shown in Fig. 13,

$$
\begin{array}{ll}
V_{u}=V_{d c}-V_{t r}-R_{t r} i_{u} & \text { if } i_{u}>0 \\
V_{u}=V_{d c}+V_{d}+R_{d}\left|i_{u}\right| & \text { if } i_{u}<0 .
\end{array}
$$

When the turn-on signal of a lower transistor 5 is active,

$$
\begin{array}{ll}
V_{u}=0-V_{d}-R_{d} i_{u} & \text { if } i_{u}>0 \\
V_{u}=0+V_{t r}+R_{t r}\left|i_{u}\right| & \text { if } i_{u}<0 .
\end{array}
$$

Here, eqs. (16), (17) and (18) do not take into account the eddy current loss, hysteresis loss, friction loss and viscous loss. The simulated result and the measured one are shown in Figs. 14 and 15. It is found that the simulated result agree well with the measured one. The stator current flows in the period of 120 degree. Even if the upper transistor turns on, the waveform of the stator voltage is not a DC voltage $V_{d c}$, but approximately same value as the electromotive force 
because of the large inverter resistance.

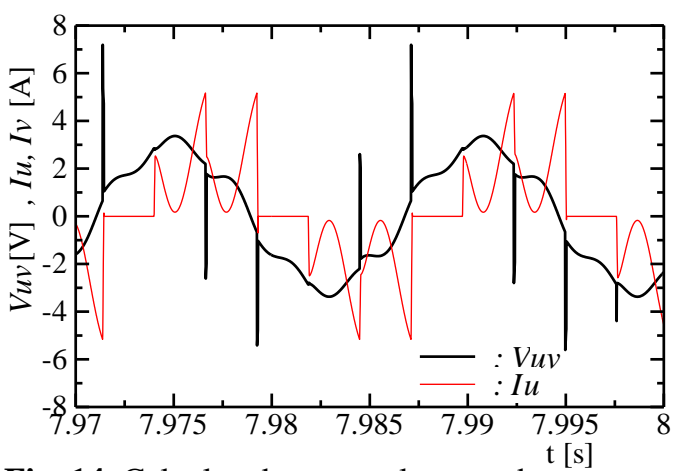

Fig. 14. Calculated stator voltage and current.

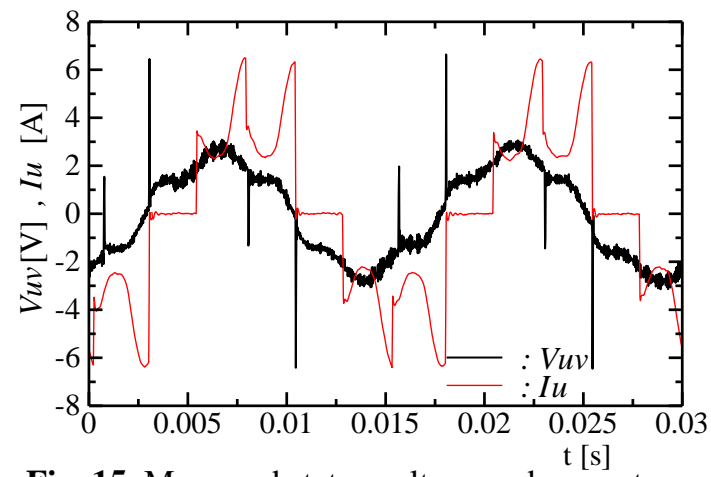

Fig. 15. Measured stator voltage and current.

\section{Conclusion}

This paper has proposed a simple equivalent circuit for the calculation method of several losses and the efficiency of brushless DC motors. The copper loss, eddy current loss, hysteresis loss, friction loss and viscous loss, and inverter loss are taken into account in the circuit. This circuit can calculate easily the maps of the motor efficiency and losses by using the Microsoft Excel, even if the motor is built in the system with a large inverter loss and/or mechanical loss.

\section{References}

[1] Y. Kawase, T. Ota, and H. Fukunaga, "3-D eddy current analysis in permanent magnet of interior permanent magnet motors," IEEE Trans. Magnetics, vol. 36, no. 4, pp. 1863 1866, Jul. 2000.

[2] A. Cassat, C. Espanet, and N. Wavre, "BLDC motor stator and rotor iron losses and thermal behavior based on lumped schemes and 3-D FEM analysis," IEEE Trans. Industrial Applications, vol. 39, no. 5, pp. 1314-1322, Sep./Oct. 2003.

[3] M. R. Shah and B. L. Sang, "Rapid analytical optimization of eddy current shield thickness for associated loss minimization in electrical machines," IEEE Trans. Industrial Applications, vol. 42, no. 3, pp. 642.649, May/Jun. 2006.

[4] K. Yamazaki, "Effect of eddy-current loss reduction by magnet segmentation in synchronous motors with concentrated windings," IEEE Trans. Industrial Applications, vol. 47, pp. 779-788, Mar./Apr. 2011.
[5] S. Morimoto, Y. Takeda, T. Hirasa, "Loss minimization control of permanent magnet Synchronous motor drives," IEEE Trans. Industry Electronics, vol. IE-41, no. 5, pp. 511$517,1994$.

[6] T. Kenjo and S. Nagamori, New brushless motor, 2000, pp. 188-203 (in Japanese).

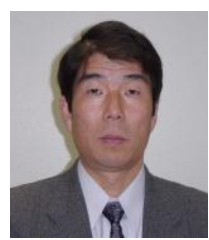

Takeo Ishikawa graduated from Tokyo Institute of Technology in 1983. He joined Gunma University in 1983, and now he is a professor. His research interests include electrical machine and power electronics. He received the 1998 best paper award of IEEE Transaction on Vehicular Technology. He is a senior member of IEEE.

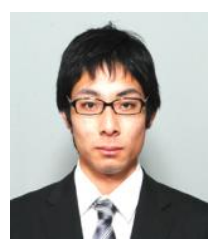

Takuma Tsuji received B.S degree in electrical and electronic engineering from Gunma University, Japan. He joined Yamada Manufacturing CO., LTD. in 2013.

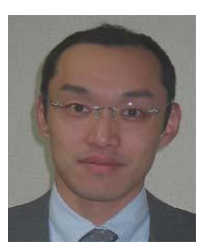

Seiji Hashimoto received the M.E. and $\mathrm{Ph} . \mathrm{D}$. degrees in Electrical and Electronic Engineering from Utsunomiya University, Tochigi, Japan, in 1996 and 1999, respectively. Since 2002, he has been a Research Associate in the Department of Electronic Engineering, Gunma University, where he is now Associate Professor. His research interests include system identification, motion control, vibration control and its application to industrial fields.

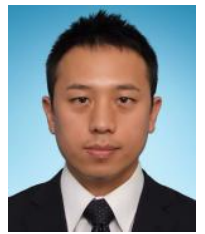

Nobuyuki Kurita received B.S degree and M.S. degree from Ibaraki University in 2001 and 2003, respectively. And he received his $\mathrm{Ph} . \mathrm{D}$. degree in engineering from Ibaraki University in 2006. He joined Gunma University as an assistant professor in 2009. His research interests include application of magnetic bearing and self-bearing motor. Dr. Nobuyuki Kurita is a member of IEEE and IEEJ. 